\title{
Identification of Risks in the Application of Surgical Robotics
}

\author{
Péter Pausits, Gábor Szögi, Marsel Nallbani, Imre J. Rudas and Tamás Haidegger \\ Óbuda University, Budapest, Hungary \\ \{peter.pausits, gabor.szogi, rudas, haidegger\}@irob.uni-obuda.hu, mnallbani@hotmail.com
}

\begin{abstract}
The continuously growing pace of robot development implies that by 2020 , there is going to be on average one robot per household in the world. The aim of robot deployment in larger numbers is to help to extend human capabilities, and to eliminate to disadvantages of the human embodiment. As a consequence, besides industrial robots, service and surgical applications appear in growing numbers. While in the field of industrial production, the adherence to sophisticated safety standards grants a low level of risk of injury, but there are numerous risks in the case of robots operating in a divided workspace. Service robots will inevitably interact physically with an operator and the served people. The complex systems of surgical robots requires risk analysis of every component, and also adequate measures to reduce the hazards. To apply these, the probable risks and their consequences have to be identified first, while making sure the robot fulfills its function.
\end{abstract}

\section{INTRODUCTION}

Contrary to robots used in industrial production, domestic robots and medical robots [1] function in divided workspace [2]. They will inevitably come into direct physical contact with people in their environment, let it be the operator or the people the robot serves. Consequently, it is hard to define the expected level of safety, as in the case of an error, minimal injuries are unavoidable [3]. However, currently operating surgical and other kinds of robots prove that their application reduces the risks during the surgical procedure and time of recovery, and increases the precision during the treatment and removal of human tissue. It is becoming evident that surgical robots will be indispensable far complex procedures in the future. In the case of certain routine procedures, like laser excisions (LR) - used to treat benign prostatic hyperplasia $(\mathrm{BPH})$ - or prostate cancer, mater-slave type robots are currently considered robust and safe. Robots functioning on the expected level of safety are complex systems containing three components: software programs, mechanics and electronics [4][5]. Every component has a number of small interfaces, of which none should be neglected. Before the final setup of a surgical robot, a highly sophisticated testing procedure is required. As opposed to industrial robots, in the case of a failure, something more than just material damage is at risk: it is human life. Currently, the most advanced system methodology is Hazard Identification and Safety Insurance Control (HISIC), which provides a guideline for robotic development, design, research, testing, application and maintenance. When can we say that a surgical robot is safe? It is a question of utmost importance, as in real life accidents and injuries can occur, and the system has to be able to handle them. Therefore safety is not just prevention of failures, but is also the most adequate way of treating a failure or injury. The goal of our work is to define the expected level of safety with the help of existing methodologies. In his paper, the necessity of HISIC is reviewed. Furthermore, the three main components from the aspect of the robot's physical build (software, mechanics and electronics), and their intersections are going to be presented.

\section{OVERVIEW OF HISIC}

HISIC is a system methodology which provides a guideline for the robotic developments from the very first researches to the design and the final product [6]. It is suggested in many cases that during the development of a surgical robot, a team with a wide range of knowledge should work together keeping the fundamental guidelines in mind. HISIC has seven basic principles. By the application of these principles the development, the design, the usage and the maintenance of the robot can get enough attention from every aspect.

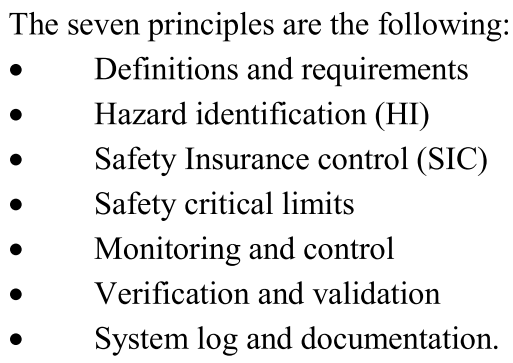

The adequate application of these principles allows the reduction of the probable risks that can occur during the operation. Hazard can be reduced to the minimal level if the operator realizes danger in time and acts right away. The task of robot does not stop at executing the operations. They also have to help the operator perceive, 
and identify the stochastic and deterministic hazards, and if needed, help them intervene at once to avoid injuries. With the help of robots, reaction time can be reduced to its fraction if the monitoring system's sampling and reaction times are suitable for the prevention of hazard or the reduction of damage caused by accidents and injuries. HISIC provides a methodology to realize this process.

\section{THE COMPLEX ROBOT CELL}

A complex robot cell, let be it industrial, surgical or service robot, is made of a number of separate components. The three principal components are electronics, mechanics and software. The components' have small interfacing from the aspect of safety; next up, these intersections and the way to eliminate them and reduce the risk of accidents is going to be presented.

Naturally, the components have to work together to execute the required operation. The lack of cooperation can also pose a threat.

\section{MECHANICS}

The primary usage of robots is in industrial environments, to increase productivity and safety. The goal is to eliminate error caused by humans. The application of complex robots increases the precision, decreases the time required, and reduces hazard by executing complex calculations. But with the application of robots, new risks came into view, that originates from the mechanical functioning of the machines. Robots expand human factors; from the aspect of safety, the two most important physical factors are carrying capacity and the increased speed of the operation. A mechanical structure significantly surpassing human abilities from the point of view of these two factors should be designed. By consequence, severe injuries can be done to the operator and the patient and the robot itself too, if it can come to contact with human body or its environment in an uncontrolled way. This risk was eliminated in the case of industrial robots by physically isolating the machines from humans in normal operating mode. But the surgical robots are different in design [7]. However, robots used in surgery make human-machine contact inevitable, as surgical procedures are performed on living organs. Prevention of injuries that can be caused by the robot is of top importance. The speed of the single operations has to be determined in a way that it can stop automatically in case of any danger, before the occurrence of an accident. The application of safety feedback sensors is indispensable for the identification of danger. Thus, the robot has to approach the patient in an optimal velocity for the execution of the operation. During the autonomous procedure, the electronic sensors have to continuously monitor the position of the tissue that is being operated on, and in the case of any unforeseen change, the robot has to be able to correct itself, or to stop. Both of these operations are only possible next to a slower pace of operation. In the master-slave type robots the doctor have to scan the tissue movements.

Surgical robot systems are usually equipped with several arms that are able to perform different operations, all of which are considered separate systems from the aspect of safety. These are able to move and perform independently, while still working in the same environment. Thus, the arms can pose danger to each other in case the control does not regulate their position compared to each other. The other advantage that robots have over human factors is precision. However, to achieve precision, the accuracy of the used sensors and driving motors is not sufficient; the physical skeleton of the robot has to be adequately stable. The potential danger that the robot poses is not the only hazard; the hazard indirectly caused by the operator is part of the safety factors. To approach accuracy, the robot has to be able to remain stable in case of a physical impact, e.g., accidental contact with a person.

The prime example of current surgical robots, the da Vinci surgical System (Sunnyvale, CA) is considered an priority stable device (Fig. 1.); how-ever, technical problems can still occur. Looking at the statistics of failures, most of them are mechanical failures of the joints and motors in $0.2-0.5 \%$ of the cases [8].

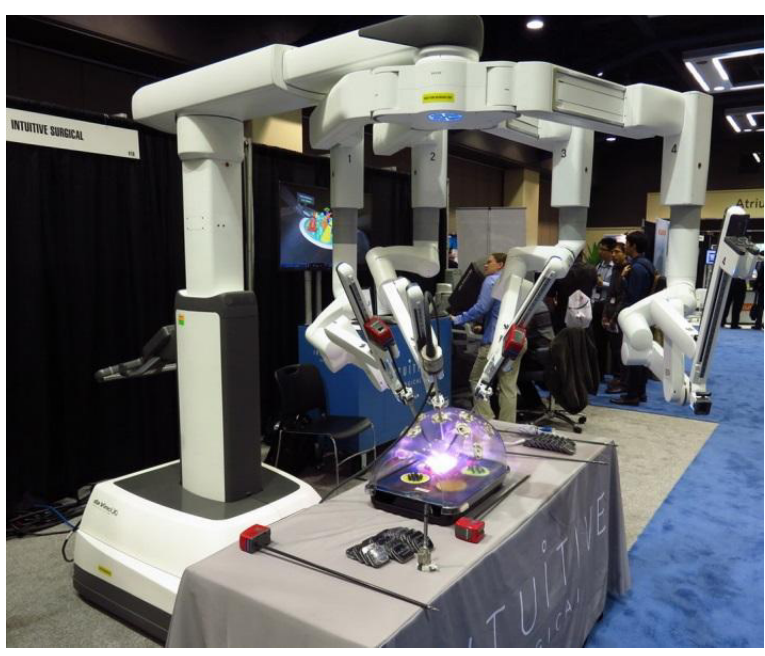

Fig. 1. The da Vinci Xi complete teleoperational surgical robot.

To sum up, a number of mechanical hazards should be taken into consideration during the design and the application of the robot, however in many cases, the management of risk should be done in the environment of robot, not in the system itself. During the application of a robot, the following mechanical hazards can occur:

- unintended movement of robot arm, axis

- material falling or ejected,

- $\quad$ sharp surfaces, edges, corners,

- dangers related to combined machines,

- unintended movement of end-effector,

- end-effector failure,

- unintended release tool.

\section{ELECTRONIC SAFETY}

In order for a robot to react as quickly as possible to the stochastic processes during a surgical procedure, the most advanced sensors, image processing capabilities and the fastest communication are necessary. Therefore, by electronic safety we mean monitoring, evaluation of the received information and regulating devices. The expected level of safety can be corrupted if the information received by the sensors is inaccurate. Therefore the application of redundancy is key in the communication of the sensors, 
and the robot and its operator. Even with the most thorough maintenance, sensors can suffer a malfunction, which can lead to a fatal error. During the creation of redundancy it is suggested to apply sensors assessing the same condition that work by different principles and have different properties. It is important as by experience, in different environments the single sensors can react differently; in ways they are not different from each other, but they are optimized for various circumstances. The information received by sensors would be evaluated by a software program. By applying such sensors the level of safety can be increased. During every surgical procedure, continuous comparison to the initial state is of top importance, so that the software program can constantly monitor every operation executed by the robot in every single moment. Furthermore, in the case of surgical robots, the body part that is being operated can change position several times, as it is problematic to completely fix human tissue in place.

High precision image processing systems are used to assess the initial state and to maintain the monitoring. The da Vinci S/Si types of surgical system, currently the most popular surgical robot, are equipped with excellent quality Full HD (high definition-1920x1080 pixels) stereo endoscopes, which can provide $3 \mathrm{D}$ vision to the operator; and which can enable precise manipulation at the tissue. Furthermore, researches are being conducted about integrated ultrasound sensors and about detecting the position of body parts in real time via 3D stereo cameras. Current research results show that ultrasound imaging is not yet suitable for continuous automated control during surgical procedures as the time delay due to it being very slow will make it hard to prevent accidents. The primary problem is with the operation of ultrasound sensor, but there are also problems with the general, non-goaloriented application of software programs. Unfortunately, imaging based on optical principles can only execute a superficial monitoring. Thus, for the long-term development of the independency of robots, a sensor that can explore the area under the surface of the human body and can monitor the position required for control is necessary. The da Vinci was not meant for automated or pre-programmed surgeries, continuous control by qualifies surgeons is required in each clinical case. This system is suitable for remote surgeries, although in this case, security issues grow in importance as inadequate communication can pose further risks. Internet is a service available worldwide, which makes it appropriate for this operation, but lot of communications protocol are exist [9].

If the robot suffers a malfunction despite the redundant detection, the operator has to intervene immediately. The most effective way of doing so is by using an emergency button that should be placed in a way that allows the quickest reach. This solution should only be used in extremis, if robot fails and can't identify and prevent the danger caused by itself. This is another reason for an expert to be present when the robot operates.

Times of industrial robots, information technology wasn't so advanced that is could have been used for the control of robots. However, after the elaboration of computers functioning by von Neumann's principles, computers and software programs started rapidly developing and spreading. Today, we could not imagine surgical and service robots without a central computer that synchronizes the single sensors and actuators, based on different pieces of information. Environmental information is provided by sensors and commands come from the operators or the automated programming. The adequate functioning of the software program is responsible for making the suitable commands; and if necessary, the program will intervene the execution of the operators' command. For example, in order to avoid possible accidents, the da Vinci immediately freezes its motion as soon as the surgeon turns their head away from the screen, or releases the control joystick [10]. During the development of the software program the developers have to keep in mind that the commands given to actuators have to remain safe for the environment, and injuries of the operators caused by mechanics in divided workspace have to be prevented.

The software is the part of a robot that assumes that the mechanics, the electronics and the communication in the robot function adequately and error-free [11]. If it ever senses an error, commands to reduce risk have to be made. In case the program cannot eliminate the problem to a sufficient extent, it will have to decide about switching the fail safe mode of robot. Every programmable unit of a robot has to be entirely stable, runtime errors are unacceptable. Thus, a robot has to go through a number of tests before set-up. It is important for the software to have the ability to overwrite and correct obviously irregular commands from the operators. However, the operators have to be able to intervene - should a malfunction occur. The most trivial way of doing so is by pressing the emergency button.

The central computer processes data using binary coding system, so information provided by the sensors must be converted to properly signals. As a consequence of the contact of the components a number of small intersections appear, as every single data conversion inevitably implies a loss of data and time delay. Environmental information is generated in the form of analog signal (temperature, pressure and movement) and sensors forward the corresponding signal. The conversion of these signals is inevitable for the central computer to process the data and make decisions accordingly.

Sensors that measure environmental information mostly use pulse-width modulation (PWM), frequency modulation (FM), amplitude modulation (AM) and discreet voltage change as signals to carry information. However, the control of actuators happens mostly using PWM signals, which implies one more conversion, and, therefore implies loss of data and time delay. During the creation of concept and selection of used components, one must strive to use sensors and actuators with the least loss of data.

The software program is accountable for the proper

TABLE I

THE MOST POPULAR SYSTEM BUS AND THEIR PROPERTIES

\begin{tabular}{|c|c|c|c|}
\hline System bus & $\begin{array}{c}\text { Data } \\
\text { transfer } \\
\text { rate } \\
\text { (kbit/s) }\end{array}$ & $\begin{array}{c}\text { Maximum } \\
\text { number of } \\
\text { communication } \\
\text { devices (pcs) }\end{array}$ & $\begin{array}{c}\text { Maximum } \\
\text { length of } \\
\text { wire (m) }\end{array}$ \\
\hline CAN Bus & $50-1000$ & 64 & $40-1000$ \\
\hline PROFI Bus & $9,6-12000$ & 32 & $100-1200$ \\
\hline LIN Bus & 20 & 16 & 40 \\
\hline LonWorks & $78-1250$ & $32385(127)$ & $125-2200$ \\
\hline INTER Bus & 500 & 4096 & $200-13000$ \\
\hline P-NET Bus & 76,8 & 125 & 1200 \\
\hline
\end{tabular}


management of the communication protocols. Usually, in such complex systems, the single components use system buses, but direct contacts exist too. When choosing the system buses the data transfer rate and the reliability have to be taken into consideration, both of which depend on the applied distance. The criteria of system buses involve the number of devices that it can connect. The following comparison presents the most popular system bus and their properties (Table I) [12][13][14].

Communication is not only important in the local network, but also in the realization of remote surgery. The da Vinci is excellently suitable for surgical procedures if the patient-side manipulator and the surgeon-side steering console are in the same room, but long-distance remote surgeries performed with this robot are only at an experimental stage. The early competition of da Vinci, the Zeus system (Computer Motion, Santa Barbara, CA), performed a successful remote surgical procedure in 2001, between America and Europe (Lindbergh Operation). Zeus used UDP (User Datagram Protokol) based communication protocol, which made it easy for the robot to connect to the internet and to overcome distance.

\section{RISK ANALYSIS}

Risk analysis is a lot more important in the case of surgical robots than in the case of industrial robots. There are many risk reducing methods [15], such as the preliminary danger analysis, the error tree analysis, the "what if..." analysis, the hazard and Operability Studies (HAZOP), the event tree analysis, the failure mode and effects analysis, the Method Organized for Systematic Analysis for Risk (MOSAR) analysis. But currently, the most advanced ones are Failure Modes Effects Analysis (FMEA) or a Failure Modes Effects and Criticality Analysis (FMECA), which are complex methods to analyze possible risks. FMEA/FMECA is presented in a table which can give an immediate answer to errors. It is based on the observable malfunction and shortly explores the possible reason behind the error and the observable consequences of the error. The expected operations to eliminate the error are necessarily included in the FMEA/FMECA table. The methodology of the FMEA operation has been recorded within the IEC 60812:2006

TABLE II

\begin{tabular}{|c|c|c|c|c|}
\hline Item & Identification & $\begin{array}{c}\text { Failure } \\
\text { modes }\end{array}$ & Effects & Safeguards \\
\hline 1. & Sensor & $\begin{array}{c}\text { Not } \\
\text { connect }\end{array}$ & $\begin{array}{c}\text { Error } \\
\text { signal }\end{array}$ & $\begin{array}{c}\text { Use secondary } \\
\text { sensor }\end{array}$ \\
\hline 2. & Communication & $\begin{array}{c}\text { Not } \\
\text { connect }\end{array}$ & $\begin{array}{c}\text { Injured } \\
\text { wire }\end{array}$ & $\begin{array}{c}\text { Mechanic } \\
\text { safety of cable }\end{array}$ \\
\hline 3. & Engine & $\begin{array}{c}\text { Not } \\
\text { move }\end{array}$ & False & $\begin{array}{c}\text { Regular } \\
\text { maintenance }\end{array}$ \\
\hline
\end{tabular}

international standard (Table II).

\section{CONCLUSION}

It can be concluded that a modern surgical robots are devices of increased complexity, and their malfunction can cause serious damage due to the workspace being shared with humans and other robots. Contrary to industrial robots, the damage is not just a material or moral disadvantage; human lives can be in danger. During the entire development of the robot, from the concept to the final testing, the design, the validation, the set-up and the maintenance, risk analysis and reduction have to be carried out with extreme caution. Understandably, numerous international standards and norms support these processes. From the aspect of safety, electronics, mechanics and the software are important components and their cooperation and co-testing are indispensable [16]. Every source of hazard has to be analyzed and resolved, as a source of risk that seems negligible can grow to pose a serious danger due to the interaction of the components. While the industrial domain is well established, novel application fields - such as medical robotics - lack the profound standardization background. Recently, various international bodies (IEC, ISO, IEEE) established study groups to better support the safe development and testing of surgical robots.

To summarize, robots functioning in shared workspace, especially surgical robots require components of the best quality and also adequate safety processes both for the development and operation.

\section{ACKNOWLEDGMENT}

T. Haidegger is a Bolyai Fellow of the Hungarian Academy of Sciences. The authors acknowledge the support of the Austrian Center for Medical Innovation and Technology (ACMIT, www.acmit.at).

\section{REFERENCES}

[1] Virk, G. S., and Haidegger, T. (2012). Classification guidelines for personal care robots-medical and non-medical applications. In Proc. of the 2012 IEEE IROS Workshop on Safety in HumanRobot Coexistence \& Interaction pp. 33-36.

[2] L. Schreiter, D. Bresolin, M. Capiluppi, J. Raczkowsky, P. Fiorini és H. Woern, "Application of Contract-based verification techniques for Hybrid Automata to Surgical Robotic Systems", 2014 European Control Conference (ECC), pp. 2310-2315.

[3] T. Kerezovic, G. Sziebig, B. Solvang and T. Latinovic, "Human Safety in Robot Applications - Review of Safety Trends", 11th International conference on accomplishments in Electrical and Mechanical Engineering and Information Technnology (DEMI 2013), pp. 1031-1039.

[4] Hoeckelmann, M., Rudas, I. J., Fiorini, P., Kirchner, F., and Haidegger, T. (2015). Current Capabilities and Development Potential in Surgical Robotics. Int J Adv Robot Syst, 12, 61.

[5] Haidegger, T., and Rudas, I. J. (2014). From Concept to Market: Surgical Robot Development. Handbook of Research on Advancements in Robotics and Mechatronics, 242

[6] B. Fei, Wan Sing NG, Chee Keong Kwoh, "The Hazard Identification and Safety Insurance Control (HISIC) for Medical Robot", 2000 22nd Annual EMBS International Conference, pp. 3022-3026.

[7] Min Yang Jung, Russell H. Taylor and P. Kazanzides, "Safety Design View: A Conceptual Framework for Systematic Understanding of Safety Features of Medical Robot Systems", 2014 IEEE International Conference on Robotics and Automation, pp. $1883-1888$

[8] T. Haidegger, "The advancement of robotic surgery - successes, failures, challenges", Orv. Hetil., 2010, 41, pp. 1690-1696.

[9] Haidegger, T., Sándor, J., and Benyó, Z. (2011). Surgery in space: the future of robotic telesurgery. Surgical endoscopy, 25(3), pp. 681-690.

[10] Jiajie Yu, Yingqiang Wang, Youping Li, Xianglian Li, Cuicui Li and Jiantong Shen, "The Safety and effectiveness of Da Vinci surgical system compared with open surgery and laparoscopic surgery: a rapid assessment", Journal of Evidence-based Medicine 7., vol. 2014. pp. 121-134. 
INES 2015 • IEEE 19th International Conference on Intelligent Engineering Systems • September 3-5, 2015, Bratislava, Slovakia

[11] P. Kazanzides, Y. Kouskoulas, A. Deguet and Z. Shao, "Proving the Correctness of Concurrent Robot Software", 2012 IEEE International Conference on Robotics and Automation, pp. 47184723.

[12] http://www.kvaser.com/about-can/can-standards/linbus/ (date: 23. April 2015)

[13] http://www.freescale.com/files/microcontrollers/doc/reports prese ntations/LINOVERVIEWPRESENT.ppt (date: 23. April 2015)

[14] Balázs Novák - Comparison of fieldbus system buses (Terepi buszrendszerek összehasonlítása) K. Elissa, "Title of paper if known," unpublished.
[15] Dr Gábor Csutorás - The science of safety (Biztonságtudomány)

[16] P. Kazanzides, G. Fichtinger, G. D. Hager, A. M. Okamura, L. L. Whitcomb and R. H. Taylor, "Surgical and Interventional Robotics", IEEE Robotics \& Automation Magazine, 2008 June, pp. 122-130. 